\title{
Estudo de Problemas de Otimização Irrestrita via Método do Gradiente
}

\author{
Marila T. Aguiar* $^{*} \quad$ Roberta M. A. Alves $^{\dagger} \quad$ Elenice W. Stiegelmeier \\ Universidade Tecnológica Federal do Paraná - UTFPR \\ Coordenação do curso de Matemática - COMAT \\ 86300-000, Campus Cornélio Procópio, PR \\ E-mail: marila@maremarseguros.com.br, roo.almeidaa@gmail.com, \\ elenicew@utfpr.edu.br.
}

\section{RESUMO}

Otimização, direta ou indiretamente, é um tema muito presente no dia a dia acadêmico e industrial. Vários campos da ciência fazem uso das ferramentas de otimização com o objetivo de ajudar na tomada de decisão. Dentre eles, pode-se citar, agricultura, finanças, transporte, processos químicos, produtivos, recursos naturais, ambientais e energéticos, entre outros [1], [6]. Nesse processo, o objetivo é minimizar os maximizar certa variável, como o custo ou o lucro em determinado processo.

Mais formalmente, pode-se dizer que otimização consiste em encontrar pontos de mínimo ou de máximo de uma função real sobre um conjunto de restrições. Isto pode ser expresso da seguinte forma:

$$
\min f(x)
$$

$$
\begin{aligned}
\text { sujeito a } & \\
g_{i}(x) & =a_{i}, \quad i=1,2, \ldots, p \\
h_{j}(x) & \geq b_{j}, \quad j=1,2, \ldots, n \\
x & \geq 0
\end{aligned}
$$

onde $x$ é um vetor n-dimensional, $f(x)$ é o funcional objetivo, $g_{i}(x)$ são restrições expressas por igualdade, $h_{j}(x)$ são restrições expressas por desigualdades e $a_{i}$ e $b_{j}$ são constantes.

Problemas de otimização são classificados conforme a forma de $f(x)$. Nesse contexto, se $f(x)$ não for linear e/ou as restrições forem não lineares, tem-se o caso da programação não linear. Além disso, quando as restrições (1) e (2) são incluídas, tem-se problemas de otimização com restrições, caso contrário, tem-se problemas de otimização sem restrições.

Neste trabalho será estudado problemas onde todas as funções usadas para definí-los são continuamente diferenciáveis e, normalmente, não lineares, isto é, o problema de programação não linear (PNL). O caso particular abordado é o problema irrestrito, ou seja, problemas de otimização sem restrições.

O problema irrestrito pode ser considerado simples, em comparação aos demais problemas de PNL, e o estudo de suas propriedades, bem como dos métodos que o resolvam, é de fundamental importância em otimização, uma vez que muitos métodos de resolução de PNL fazem uso dos métodos que resolvem o caso irrestrito [2].

Para a obtenção da solução de um problema como o descrito acima, pode-se utilizar uma condição necessária de otimalidade, a qual permite a obtenção de uma candidata à solução analítica para o problema e, portanto, exata. Porém, em muitos casos, se torna difícil e até mesmo impraticável, obter uma candidata a solução analiticamente devido à complexidade das expressões envolvidas. Em casos como

\footnotetext{
*bolsista de Iniciação Científica UTFPR

† bolsista de Iniciação Científica UTFPR
} 
esses, precisa-se utilizar métodos numéricos, com os quais é possível a obtenção de uma solução aproximada. Neste trabalho será estudado algumas situações que garantem a existência de um minimizador e, em seguida, é discutido as condições de otimalidade para o problema de otimização irrestrita. Algumas referências para este assunto são [3], [5], [6] e [7].

Para a resolução numérica do problema de PNL irrestrito será utilizada uma das técnicas mais conhecidas para minimizar uma função, o método clássico do gradiente, também chamado método de Cauchy. O método do gradiente é um processo iterativo que a cada etapa faz uma busca na direção do vetor gradiente da função objetivo no ponto corrente [4] e consiste em procurar o mínimo na direção de maior taxa de decrescimento da função objetivo a partir de uma solução inicial $x_{0}$.

Como o nome implica, os métodos gradientes usam explicitamente informação sobre a derivada para gerar um algoritmo eficiente para localizar o ponto ótimo. Uma das vantagens de métodos do tipo gradiente é que a informação da derivada da função permite alcançar o extremo com menor número de avaliações da função, ou seja, melhor eficiência computacional.

Assim, o método gradiente requer como entrada uma solução inicial $x_{0}$ e um procedimento que calcula o gradiente da função a ser maximizada (ou minimizada). Basicamente, o algoritmo pode ser descrito como um processo iterativo em que novos pontos $x_{k+1}$ são gerados a partir do ponto atual $x_{k}$ :

$$
x_{k+1}=x_{k}+\epsilon_{k} \nabla f\left(x_{k}\right)
$$

onde $\nabla f\left(x_{k}\right)$ é a direção de busca linear, $\epsilon_{k}$ representa o tamanho do passo a ser dado na direção de busca e $k$ é o número de iterações.

O algoritmo foi implementado em linguagem de programação com apoio do software matemático MatLab. No algoritmo implementado, a determinação do tamanho do passo $\epsilon_{k}$ é deixado em aberto. Para validar os resultados, foram feitas comparações de diversos problemas de PNL da literatura, usando como método de resolução o método do gradiente e o Optimization Toolbox do MatLab, o qual é uma coleção de funções que estendem a capacidade do Matlab em resolver problemas de otimização.

Portanto, este trabalho apresenta o desenvolvimento teórico das condições de otimalidade para problemas de otimização, bem como, o estudo de métodos iterativos para obter as soluções numéricas.

Palavras-chave: Otimização, programação não linear, método do gradiente.

\section{Referências}

[1] M. Arenales, V. Armentano, R. Morabito, H. Yanasse, "Pesquisa Operacional”, Elsevier, Rio de Janeiro, RJ, 2007.

[2] D. P. Bertsekas, "Nonlinear Programming”, Athena Scientific, Belmont, MA, 1995.

[3] F. H. Clarke, Yu. S. Ledyaev, R. J. Stern, P. R. Wonlenski, "Nonsmooth Analysis and Control Theory", Series Graduate texts in mathematics, Springer-Verlag, New York, 1998.

[4] S. C. Chapra, R.P. Canale, "Métodos Numéricos para Engenharia", MacGraw-Hill, São Paulo, 2008.

[5] A. Izmailov, M. Solodov, "Otimização: condições de otimalidade, elementos de análise convexa e dualidade", v.1, IMPA, Rio de Janeiro, 2005.

[6] A. A. Ribeiro, E.W. Karas, "Otimização Contínua: Aspectos Teóricos e Computacionais”, Cengage Learning, São Paulo, 2013.

[7] R. Vinter, "Optimal Control”, Birkhauser, New York, NY, 2000. 